\title{
CUORE crystal validation runs: results on radioactive contamination and extrapolation to CUORE background
}

F. Alessandria ${ }^{\mathrm{a}}$, E. Andreotti ${ }^{\mathrm{b}, \mathrm{c}, 1}$, R. Ardito ${ }^{\mathrm{d}}$, C. Arnaboldie, F. T. Avignone III ${ }^{\mathrm{f}}$, M. Balata ${ }^{\mathrm{g}}$, I. Bandac ${ }^{\mathrm{f}}$, T. I. Banks ${ }^{\mathrm{h}, \mathrm{i}, \mathrm{g}}$, G. Bari $^{\mathrm{j}}$, J. W. Beeman ${ }^{\mathrm{k}}$, F. Bellini ${ }^{\mathrm{l}, \mathrm{m}}$, A. Bersani ${ }^{\mathrm{n}}$, M. Biassoni ${ }^{\mathrm{e}, \mathrm{c}}$, T. Bloxham ${ }^{\mathrm{i}}$, C. Brofferio $^{\mathrm{e}, \mathrm{c}}$, A. Bryant $^{\mathrm{i}, \mathrm{h}}$, C. Bucci $^{\mathrm{g}}$, X. Z. Cai $^{\mathrm{o}}$,

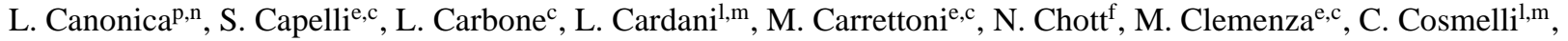
O. Cremonesi ${ }^{c}$, R. J. Creswick ${ }^{\mathrm{f}}$, I. Dafinei ${ }^{\mathrm{m}}$, A. Dally ${ }^{\mathrm{q}}$, A. De Biasi ${ }^{\mathrm{r}}$, M. P. Decowski ${ }^{\mathrm{i}, \mathrm{h}, 2}$, M. M. Deninno ${ }^{\mathrm{j}}$, A. de Waard ${ }^{\mathrm{s}}$,

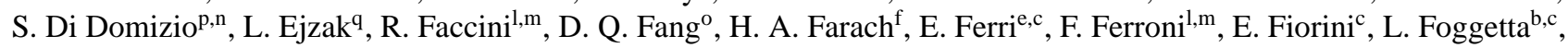
S. J. Freedman ${ }^{\mathrm{i}, \mathrm{h}}$, G. Frossati ${ }^{\mathrm{s}}$, A. Giachero ${ }^{\mathrm{c}}$, L. Gironi ${ }^{\mathrm{e}, \mathrm{c}}$, A. Giuliani ${ }^{\mathrm{t}}$, P. Gorla $^{\mathrm{u}}$, C. Gotti ${ }^{\mathrm{e}, \mathrm{c}}$, E. Guardincerri $^{\mathrm{g}, \mathrm{i}}$, T. D. Gutierrez ${ }^{\mathrm{v}}$, E. E. Haller ${ }^{\mathrm{k}, \mathrm{w}}$, K. Han ${ }^{\mathrm{i}}$, K. M. Heeger ${ }^{\mathrm{q}}$, H. Z. Huang ${ }^{\mathrm{x}}$, K. Ichimura ${ }^{\mathrm{i}}$, R. Kadel ${ }^{\mathrm{y}}$, K. Kazkaz ${ }^{\mathrm{z}}$, G. Keppel ${ }^{\mathrm{r}}$ L. Kogler $^{\mathrm{i}, \mathrm{h}}$,

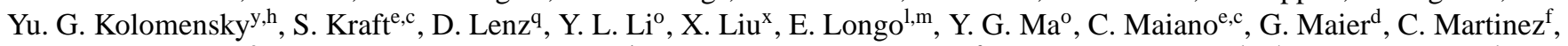

M. Martinez ${ }^{\text {c,aa, } 3}$, R. H. Maruyama ${ }^{\mathrm{q}}$, N. Moggi ${ }^{\mathrm{j}}, \mathrm{S}$. Morganti ${ }^{\mathrm{m}}$, S. Newman ${ }^{\mathrm{f}, \mathrm{g}}, \mathrm{S}$. Nisi ${ }^{\mathrm{g}}$, C. Nones ${ }^{\mathrm{b}, \mathrm{c}, 4}$, E. B. Norman ${ }^{\text {z,ab }}$,

A. Nucciotti ${ }^{\mathrm{e}, \mathrm{c}}$, F. Orio ${ }^{\mathrm{m}}$, D. Orlandi ${ }^{\mathrm{g}}$, J. Ouellet ${ }^{\mathrm{i}, \mathrm{h}}$, M. Pallavicini $^{\mathrm{p}, \mathrm{n}}$, V. Palmieri ${ }^{\mathrm{r}}$, L. Pattavina $^{\mathrm{c}}$, M. Pavan $^{\mathrm{e}, \mathrm{c}}$, M. Pedretti $^{\mathrm{z}}$,

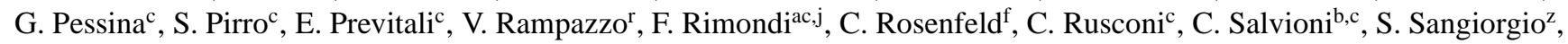

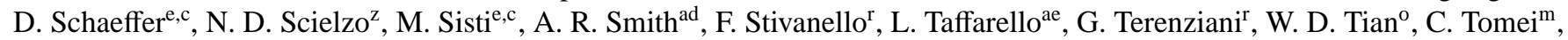

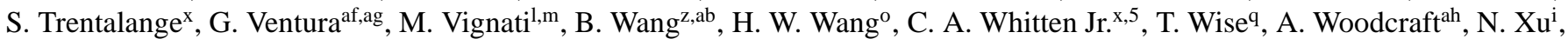
L. Zanotti ${ }^{\mathrm{e}, \mathrm{c}}$, C. Zarra ${ }^{\mathrm{g}}$, B. X. Zhu ${ }^{\mathrm{x}}$, S. Zucchelli ${ }^{\mathrm{ac}, \mathrm{j}}$

${ }^{a}$ INFN - Sezione di Milano, Milano I-20133 - Italy

${ }^{b}$ Dipartimento di Fisica e Matematica, Università dell'Insubria, Como I-22100 - Italy ${ }^{c}$ INFN - Sezione di Milano Bicocca, Milano I-20126 - Italy

${ }^{d}$ Dipartimento di Ingegneria Strutturale, Politecnico di Milano, Milano I-20133 - Italy ${ }^{e}$ Dipartimento di Fisica, Università di Milano-Bicocca, Milano I-20126 - Italy

${ }^{f}$ Department of Physics and Astronomy, University of South Carolina, Columbia, SC 29208 - USA ${ }^{g}$ INFN - Laboratori Nazionali del Gran Sasso, Assergi (L'Aquila) I-67010 - Italy

${ }^{h}$ Department of Physics, University of California, Berkeley, CA 94720 - USA

${ }^{i}$ Nuclear Science Division, Lawrence Berkeley National Laboratory, Berkeley, CA 94720 - USA ${ }^{j}$ INFN - Sezione di Bologna, Bologna I-40127 - Italy

${ }^{k}$ Materials Science Division, Lawrence Berkeley National Laboratory, Berkeley, CA 94720 - USA

${ }^{l}$ Dipartimento di Fisica, Università degli Studio di Roma "La Sapienza”, Roma I-00185 - Italy ${ }^{m}$ INFN - Sezione di Roma, Roma I-00185 - Italy ${ }^{n}$ INFN - Sezione di Genova, Genova I-16146 - Italy

${ }^{o}$ Shanghai Institute of Applied Physics (Chinese Academy of Sciences), Shanghai 201800 - China ${ }^{p}$ Dipartimento di Fisica, Università di Genova, Genova I-16146 - Italy

${ }^{q}$ Department of Physics, University of Wisconsin, Madison, WI 53706 - USA

${ }^{r}$ INFN - Laboratori Nazionali di Legnaro, Legnaro (Padova) I-35020 - Italy

${ }^{s}$ Kamerlingh Onnes Laboratorium, Leiden University, Leiden, RA 2300 - The Netherlands

${ }^{t}$ Centre de Spectrométrie Nucléaire et de Spectrométrie de Masse, 91405 Orsay Campus - France ${ }^{u}$ INFN - Sezione di Roma Tor Vergata, Roma I-00133 - Italy

${ }^{v}$ Physics Department, California Polytechnic State University, San Luis Obispo, CA 93407 - USA ${ }^{w}$ Department of Materials Science and Engineering, University of California, Berkeley, CA 94720 - USA

${ }^{x}$ Department of Physics and Astronomy, University of California, Los Angeles, CA 90095 - USA

${ }^{y}$ Physics Division, Lawrence Berkeley National Laboratory, Berkeley, CA 94720 - USA

${ }^{z}$ Lawrence Livermore National Laboratory, Livermore, CA 94550 - USA

${ }^{a}$ Laboratorio de Fisica Nuclear y Astroparticulas, Universidad de Zaragoza, Zaragoza 50009 - Spain

${ }^{a b}$ Department of Nuclear Engineering, University of California, Berkeley, CA 94720 - USA

${ }^{a c}$ Dipartimento di Fisica, Università di Bologna, Bologna I-40127 - Italy

${ }^{a d}$ EHES Division, Lawrence Berkeley National Laboratory, Berkeley, CA 94720 - USA ae INFN - Sezione di Padova, Padova I-35131 - Italy

${ }^{a f}$ Dipartimento di Fisica, Università di Firenze, Firenze I-50125 - Italy ${ }^{a g}$ INFN - Sezione di Firenze, Firenze I-50125 - Italy

${ }^{a h}$ SUPA, Institute for Astronomy, University of Edinburgh, Blackford Hill, Edinburgh EH9 $3 H J$ - UK

\begin{abstract}
The CUORE Crystal Validation Runs (CCVRs) have been carried out since the end of 2008 at the Gran Sasso National Laboratories, in order to test the performances and the radiopurity of the $\mathrm{TeO}_{2}$ crystals produced at SICCAS (Shanghai Institute of Ceramics, Chinese Academy of Sciences) for the CUORE experiment. In this work the results of the first 5 validation runs are presented. Results have been obtained for bulk contaminations and surface contaminations from several nuclides. An extrapolation to the CUORE background has been performed.
\end{abstract}




\section{Introduction}

The production of the CUORE [1] crystals was appointed to SICCAS (Shanghai Institute of Ceramics, Chinese Academy of Sciences) and began in March 2008, with the synthesis of the $\mathrm{TeO}_{2}$ powder. Given the goal of CUORE in terms of background $(<0.01$ counts $/ \mathrm{keV} / \mathrm{kg} / \mathrm{y}$ at the Q-value [2]), the radiopurity of the $\mathrm{TeO}_{2}$ crystals is a crucial issue.

Radioactive contaminations may come from long-lived, naturally occurring isotopes, such as ${ }^{238} \mathrm{U}$ and ${ }^{232} \mathrm{Th}$ and their daughters and from cosmogenic activation of the detector materials after their production. To minimize the influence of longlived nuclei, great care is devoted to the selection of all materials and ancillaries used for the preparation of the detector. Sea level transport and underground storage of prepared crystals are ${ }_{47}^{46}$ necessary in order to minimize their cosmogenic activation.

A dedicated protocol [3] is defined for the radio-purity re- ${ }^{48}$ lated quality control of the crystal production process, start- ${ }^{49}$ ing from metallic tellurium synthesis to the final processing ${ }_{51}^{50}$ of ready-to-use $\mathrm{TeO}_{2}$ crystals. Radio-purity certification pro- ${ }^{51}$ cedures, involving ICP-MS (Inductively Coupled Plasma Mass ${ }^{52}$ Spectrometry) measurements, $\gamma$ spectroscopy with HPGe detectors and $\alpha$ spectroscopy with Surface Barriers Detectors (SBD), are applied in each production phase to test the above ${ }_{54}$ mentioned materials.

At the same time, cryogenic tests are designed to test the 55 ready-to-use $\mathrm{TeO}_{2}$ crystals upon their arrival at LNGS. The 56 crystal validation is performed through experimental runs, each ${ }^{57}$ called CCVR (CUORE Crystal Validation Run), in which 458 crystals randomly chosen from a batch coming from SICCAS, 59 are mounted in an setup similar to a CUORE single module and 60 operated at cryogenic temperatures for a time period of several 61 weeks in order to test the bolometric performance and the com- 62 pliance of the crystals to the contract limits in terms of radio- 63 purity (see Table 1).

\begin{tabular}{|c|c|}
\hline Isotope & Allowed Contamination \\
\hline \hline${ }^{238} \mathrm{U}$ & $<3 \cdot 10^{-13} \mathrm{~g} / \mathrm{g}$ \\
\hline${ }^{232} \mathrm{Th}$ & $<3 \cdot 10^{-13} \mathrm{~g} / \mathrm{g}$ \\
\hline${ }^{210} \mathrm{~Pb}$ & $<1 \cdot 10^{-5} \mathrm{~Bq} / \mathrm{kg}$ \\
\hline${ }^{210} \mathrm{Po}$ & $<0.1 \mathrm{~Bq} / \mathrm{kg}$ \\
\hline
\end{tabular}

Table 1: Contamination limits for the ready-to-use $\mathrm{TeO}_{2}$ crystals [3].

In this work the results of the first 5 validation runs, carried out from the end of 2008 to the middle of 2010, are presented. A summary of all CCVRs detector operation is reported in Ta- ${ }^{76}$ ble 2. The 4 crystals tested in CCVR1 (two of them were again ${ }^{77}$ tested in CCVR2) were sent to LNGS by plane. This was neces- ${ }^{7}$ sary in order to ensure a fast response on the radioactivity level of the crystals. All the other crystals were transported by ship. 79

\footnotetext{
${ }^{1}$ Presently at: Joint Research Center, Institute for Reference Materials and 80 Measurements, 2440 Geel - Belgium

${ }^{2}$ Presently at: Nikhef, 1098 XG Amsterdam - The Netherlands

${ }^{3}$ Presently at: Institut d'Astrophysique Spatial, 91045 Orsay - France

${ }^{4}$ Presently at: CEA / Saclay, 91191 Gif-sur-Yvette - France

${ }^{5}$ Deceased
}

\begin{tabular}{|c|c|c|}
\hline Run & Duration & Livetime [d] \\
\hline \hline CCVR1 & Dec 20, 2008 - Mar 9, 2009 & 59.9 \\
CCVR2 & Jun 6, 2009 - Jun 30, 2009 & 19.4 \\
CCVR3 & Nov 11, 2009 - Jan 4, 2010 & 43.05 \\
CCVR4 & Mar 31, 2010 - May 17, 2010 & 25.8 \\
CCVR5 & Aug 11, 2010 - Oct 1, 2010 & 30.3 \\
\hline
\end{tabular}

Table 2: Summary of CCVR data taking period and live time.

Radioactivity study was performed on the high energy region of the spectrum (above $4000 \mathrm{keV}$ ), where the contribution of the $\alpha$ lines from uranium and thorium decay chains is expected. Given the short range of $\alpha$ particles, their signature is a clear indication of a radioactive contamination within the crystals or on their surface. Results (mainly upper limits) are obtained for both bulk contaminations (Sec. 5) and surface contaminations (Sec. 7). For ${ }^{210} \mathrm{~Pb}$, for which the study of the $\alpha$ lines was not possible, the lower energy portion of the spectrum has been used (Sec. 6).

An extrapolation to the CUORE background is performed in Sec. 8 .

\section{Experimental setup}

Each CCVR setup consists of an array of four crystals, arranged in a single floor which in a first approximation represents the single CUORE module. The four $5 \times 5 \times 5 \mathrm{~cm}^{3}$ crystals are enclosed in a pair of Oxygen Free High Conductivity (OFHC) copper frames which serve both as mechanical support and thermal bath. The two frames are connected to each other by four small columns, also made of copper. Both frames and copper are wrapped with several layers of polyethylene. The crystals are connected to the copper frames by small Teflon supports that represent the weak thermal conductance versus the heat sink.

Each crystal is provided with a Neutron Transmutation Doped Ge thermistor (NTD), which converts the phonon signal into a detectable voltage pulse [4]. Some of the CCVR crystals are provided with two NTD thermistors. In these cases both channels are analyzed and the best performing channel (from the point of view of the energy resolution) is used for the final results.

The CCVR experimental setup is hosted in a dilution refrigerator placed in the Hall C of National Laboratory of INFN at Gran Sasso and operated at $\sim 10 \mathrm{mK}$. A complete description can be found in [5] and references therein for what concerns the electronics and the DAQ and in [6] for what concerns the cryogenic setup and the shields.

\section{Data analysis}

CCVR data processing, from raw data to the final spectra, follows the procedure described in details in [7].

The pulse amplitude is estimated by means of an Optimum Filter (OF) technique [8] that reduces the noise superimposed to the signal, maximizing the signal to noise ratio. 
The mean detector response and the noise power spectral ${ }_{127}$ density, needed to build the OF transfer function, are esti-128 mated from bolometric pulses and noise samples (data samples ${ }_{129}$ recorded randomly and without triggered events) by a proper ${ }_{130}$ averaging procedure.

Gain instability corrections [7] are performed using the ${ }_{132}$ $5407.5 \mathrm{keV} \alpha$ line from ${ }^{210} \mathrm{Po}$. This element is always present ${ }_{133}$ in recently grown crystals but decays away with a half life of ${ }_{134}$ 138.38 days.

The energy calibration is performed using ${ }^{232} \mathrm{Th} \gamma$ sources inserted inside the cryostat external lead shield. An example of ${ }^{136}$ calibration spectrum (Channel2 - CCVR1) is shown in Fig. 1$]^{137}$ Gamma lines from the ${ }^{232} \mathrm{Th}$ decay chain are clearly visible in ${ }^{138}$ the spectrum. The calibration is performed using a third-order ${ }^{139}$ polynomial function and the ${ }^{210}$ Po peak is used in addition to ${ }^{140}$ the ${ }^{232}$ Th $\gamma$ peaks.

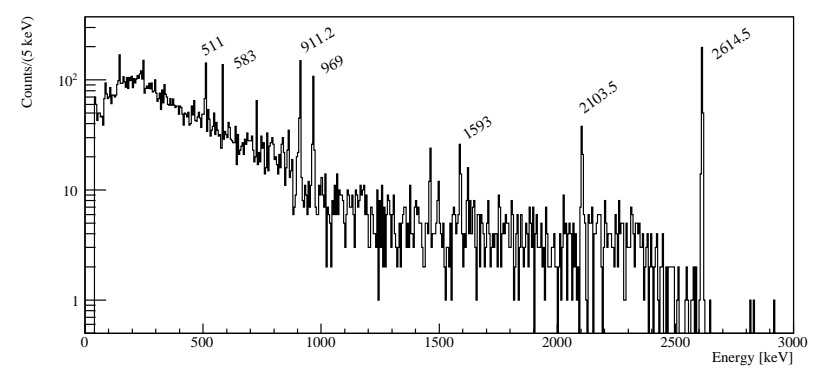

Figure 1: Calibration spectrum of Channel2 - CCVR1. Gamma lines from the ${ }^{232}$ Th decay chain are visible in the spectrum.

Each CCVR sum spectrum is composed by events which survive two different type of data selection: global and event-based requirements.

Global requirements are applied following criteria decided a priori on the detector performances (an excessive noise level, ADC saturation, etc..). They identify bad time intervals that need to be discarded. These kind of cuts introduce a dead time that is accounted for by properly reducing the live time of the interested detector.

Event-based requirements comprise: pile-up rejection, pulseshape and coincidence selection.

The presence of a pile-up prevents the OF algorithm from providing a correct evaluation of the pulse amplitude. The pileup rejection is performed by imposing an extendable (paralyzable) dead window of 7 seconds to each event.

The pulse-shape analysis is used to reject non-physical events. The pulse shape parameters are the rise time and decay time of the OF-filtered waveform and other parameters that measure the deviation of filtered raw signal from the average detector response.

As a first step, each CCVR spectrum is corrected for the corresponding efficiency of the event-based cuts (from Table 3) and ${ }^{149}$ then the spectra are summed together. Four types of spectra are ${ }_{150}$ produced for all CCVRs:

- Total Energy spectrum (TOT): it contains all the general cuts and the pulse shape cuts.
- Anti-coincidence Energy spectrum (M1): it contains the events that caused an energy deposition in one crystal only (anti-coincidence cut). For what concerns $\alpha$ particles, this corresponds to bulk events (that is decays where the emitted $\alpha$ particle is absorbed within the crystal itself) and to surface events, generated by decays occurring on the surface of an inert material that hit a facing crystal, or to $\alpha$ decays occurring on a crystal surface, whose escaping products are absorbed by inert materials.

- Coincidence Energy spectrum (M2): it contains the events that caused an energy deposition in two crystals. For what concerns $\alpha$ particles, these events arise from $\alpha$ decays occurring on a crystal surface faced to another crystal.

- Coincidence Sum Energy spectrum (M2sum): it contains the sum energy of multiplicity 2 events. For instance in the case of an $\alpha$ decay on the surface of a crystal, both the energy of the $\alpha$ particle $E_{1}$ in the facing crystal and the corresponding nuclear recoil energy $E_{2}$ in the original crystal are detected. In the spectrum M2sum the variable $E_{T O T}=E_{1}+E_{2}$ is plotted.

Fig. 2] shows the sum spectra of all CCVRs.
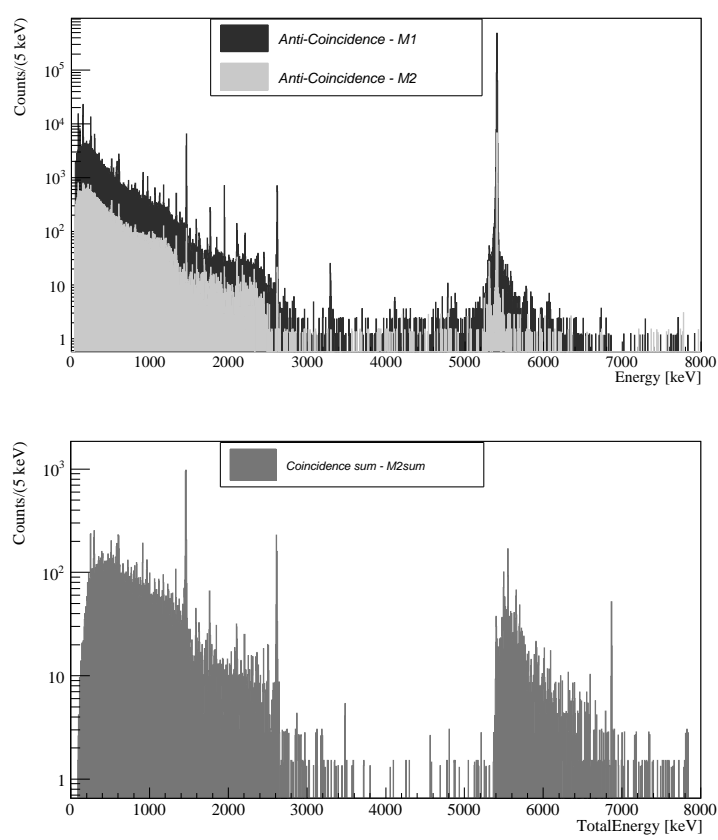

Figure 2: Energy spectra for the full CCVR statistics. Top: anti-coincidence and coincidence spectra. Bottom: Sum energy spectrum of multiplicity 2 events.

\subsection{Efficiency of event-based cuts}

Due to the high rate of ${ }^{210} \mathrm{Po}$ events, there is a significant loss of efficiency due to pile-up rejection. The efficiency is estimated as:

$$
\varepsilon_{\text {pile-up }}=1-P_{\text {pile-up }}=e^{(-r \cdot T)}
$$


where $P$ is the probability of a pile-up, $r$ is the counting rate of 199 the events that passed the global cuts described above, and $T$ is200 the length of a time interval containing an event during which 201 the occurrence of another event would be considered pile-up.202 The interval $T$ contains some time after the event during which a double pulse would result and some time before the event ${ }^{203}$ during which the event's baseline would be spoiled by the tail ${ }^{204}$ of the preceding pulse. Properly, $T$ depends on the energy of ${ }_{205}$ the other event: the higher the energy of the other event, the ${ }_{200}$ longer its tail remains too large. The counting-rate is channeldependent and sometimes also time-dependent. In the specific ${ }^{207}$ case of ${ }^{210} \mathrm{Po}$ events, the counting-rate obviously decreases with ${ }^{208}$ time due to polonium decay (half-life: 138.38 days).

For the sake of simplicity, an average pile-up reduction ef-209 ficiency for each CCVR is computed. This will apply to all channels in the full energy range and is calculated using formula (11) with $T=7 \mathrm{~s}$ and using as $r$ the global counting rate ${ }^{210}$ after the general cuts.

\begin{tabular}{|c|c|c|c|}
\hline Run & $\varepsilon_{\text {pile-up }}$ & $\varepsilon_{\text {PSA }}$ & $\varepsilon_{\text {AC }}$ \\
\hline \hline CCVR1 & $0.84 \pm 0.01$ & $0.96 \pm 0.01$ & $0.987 \pm 0.003$ \\
CCVR2 & $0.88 \pm 0.01$ & $0.98 \pm 0.01$ & $0.982 \pm 0.005$ \\
CCVR3 & $0.89 \pm 0.01$ & $0.97 \pm 0.01$ & $0.990 \pm 0.002$ \\
CCVR4 & $0.88 \pm 0.01$ & $0.94 \pm 0.02$ & $0.987 \pm 0.003$ \\
CCVR5 & $0.89 \pm 0.01$ & $0.98 \pm 0.01$ & $0.990 \pm 0.003$ \\
\hline
\end{tabular}

Table 3: Efficiencies of event-based cuts for each CCVR.

The pulse shape cuts efficiency $\varepsilon_{\text {PSA }}$ is evaluated on the background peak at $2614.5 \mathrm{keV}$ due to ${ }^{208} \mathrm{Tl}$, by a simultaneous fit on both the spectra of accepted and rejected events as detailed in [7].

The same procedure is applied for the evaluation of the anticoincidence cut efficiency $\varepsilon_{\mathrm{AC}}$. In this case, instead of the $2614.5 \mathrm{keV}$ line (which is usually in coincidence with other $\gamma$ lines), the photopeak at $1460.8 \mathrm{keV}$ due to ${ }^{40} \mathrm{~K}$ is used. Results are summarized in Table 3

\subsection{Monte Carlo simulations}

In order to extract numerical information on the activity ${ }_{216}$ or contamination of a given nuclide in CUORE crystals from 217 CCVRs data, it is necessary to rely on Monte Carlo simula-218 tions, capable of reproducing the main features of the detector ${ }_{219}$ geometry and response.

CCVRs simulations are performed with the GEANT4-based 221 code developed by the CUORICINO ad CUORE collaboration 222 and described in [9] and [10]. The simulation takes into ac-223 count the energy resolution and the threshold of each detector.224 For bulk contaminations, an homogeneous distribution of the 225 given nuclide inside the crystals is assumed. For surface con-226 taminations, different depths of the contamination layer (from 227 $0.01 \mu \mathrm{m}$ to $10 \mu \mathrm{m}$ ) are considered and the contamination den-228 sity profile is assumed to decrease exponentially with the depth ${ }_{229}$ from the surface to the bulk of the crystal.

In CCVR1 there is a not negligible number of measurements231 $(\sim 4 \%)$ with only 2 active facing crystals. This affects the effi-232 ciency calculation of surface contaminations. To take into account this variation from the standard configuration with 4 crystals, 2 different efficiencies are calculated for each Monte Carlo simulation:

- $\varepsilon_{M C}^{2 c h}$ : corresponding to the CCVR1 configuration with only 2 active crystals;

- $\varepsilon_{M C}^{4 c h}$ : corresponding to the standard CCVR configuration, with 4 active crystals;

The average efficiency, weighed on the proper lifetime $\Delta t^{i}$, is computed using the formula:

$$
\varepsilon_{M C}=\frac{\varepsilon_{M C}^{2 c h} \Delta t^{2 c h}+\varepsilon_{M C}^{4 c h} \Delta t^{4 c h}}{\Delta t^{2 c h}+\Delta t^{4 c h}}
$$

\section{Results on background rates of CUORE crystals}

From the energy spectra of all CCVRs the background rates in various energy regions can be calculated.

Six energy regions of interest are identified in the spectra and the corresponding count rates for anticoincidence (M1) and coincidence (M2) spectra are calculated. Results for the global spectra are reported in Table4(errors are statistical only).

\begin{tabular}{|c|c|c|c|}
\hline & $\begin{array}{c}\text { Continuum } \\
(2700,3200) \\
{[\mathrm{keV}]}\end{array}$ & $\begin{array}{c}{ }^{190} \mathrm{Pt} \\
(3200,3400) \\
{[\mathrm{keV}]}\end{array}$ & $\begin{array}{c}\text { Continuum } \\
(3400,3900) \\
{[\mathrm{keV}]}\end{array}$ \\
\hline M1 & $0.19 \pm 0.02$ & $0.38 \pm 0.04$ & $0.09 \pm 0.01$ \\
M1-PoSub & $0.13 \pm 0.02$ & $0.34 \pm 0.04$ & $0.06 \pm 0.01$ \\
\hline M2 & $0.05 \pm 0.01$ & $0.02 \pm 0.01$ & $0.025 \pm 0.006$ \\
M2-PoSub & $0.02 \pm 0.01$ & $0.01 \pm 0.01$ & $0.008 \pm 0.008$ \\
\hline \hline & $\mathrm{U} / \mathrm{Th}$ & ${ }^{210} \mathrm{Po}$ & $\mathrm{U} / \mathrm{Th}$ \\
& $(4000,5000)$ & $(5000,6000)$ & $(6000,8000)$ \\
& {$[\mathrm{keV}]$} & {$[\mathrm{keV}]$} & {$[\mathrm{keV}]$} \\
\hline M1 & $0.19 \pm 0.01$ & - & $0.057 \pm 0.004$ \\
M1-PoSub & $0.13 \pm 0.01$ & - & $0.057 \pm 0.004$ \\
\hline M2 & $0.04 \pm 0.01$ & - & $0.014 \pm 0.002$ \\
M2-PoSub & $0.014 \pm 0.007$ & - & $0.014 \pm 0.002$ \\
\hline
\end{tabular}

Table 4: Count rates measured in [counts $/ \mathrm{keV} / \mathrm{kg} / \mathrm{y}$ ]. Errors are statistical.

The continuum region $(2700,3200) \mathrm{keV}$ is of great interest since it is the region immediately above the Q-value of the neutrinoless double beta decay of ${ }^{130} \mathrm{Te}$ [7].

In the region $(3200,3400) \mathrm{keV}$ a contribution of the $\alpha$ line from ${ }^{190} \mathrm{Pt}$ is expected. This contamination is almost unavoidable for $\mathrm{TeO}_{2}$ crystals, as explained in Sec. 5 .

From 4000 to $8000 \mathrm{keV}$ the contribution of the various $\alpha$ lines from $\mathrm{U}$ and $\mathrm{Th}$ decay chains is expected.

In between, there is the region $(5000,6000) \mathrm{keV}$, which is affected by the ${ }^{210}$ Po contamination (see Sec. 5.1). This produces not only a peak at the energy of the Po $\alpha$ line 5407.5 $\mathrm{keV}$ but also a broad background over the entire region due to mis-identified pile-up events (above the peak energy) or to the escape of the $\alpha$ that releases part of its energy in a inert material (below the peak energy). An indication of the rate in this region is of no particular interest, also because of the relative short 


\begin{tabular}{|c|c|c|c|}
\hline & & $\begin{array}{c}\text { Continuum } \\
(2700,3900) \\
{[\mathrm{keV}]}\end{array}$ & $\begin{array}{c}\mathrm{U} / \mathrm{Th} \\
(4000,5000) \\
{[\mathrm{keV}]}\end{array}$ \\
\hline CCVR & M1 & $0.09 \pm 0.02$ & $0.13 \pm 0.01$ \\
& M2 & $0.015 \pm 0.007$ & $0.014 \pm 0.003$ \\
\hline TTT & M1 & $0.052 \pm 0.008$ & $0.28 \pm 0.02$ \\
& M2 & $0.009 \pm 0.003$ & $0.0018 \pm 0.005$ \\
\hline Cuoricino & M1 & $0.104 \pm 0.002$ & $0.522 \pm 0.003$ \\
& M2 & $0.009 \pm 0.001$ & $0.084 \pm 0.001$ \\
\hline \hline & & ${ }^{210} \mathrm{Po}$ & $\mathrm{U} / \mathrm{Th}$ \\
& & $(5000,6000)$ & $(6000,8000)$ \\
& & {$[\mathrm{keV}]$} & {$[\mathrm{keV}]$} \\
\hline CCVR & M1 & - & $0.057 \pm 0.004$ \\
& M2 & - & $0.014 \pm 0.002$ \\
\hline TTT & M1 & $1.30 \pm 0.07$ & $0.025 \pm 0.004$ \\
& M2 & $0.09 \pm 0.01$ & $0.005 \pm 0.002$ \\
\hline Cuoricino & M1 & $0.846 \pm 0.004$ & $0.099 \pm 0.001$ \\
& M2 & $0.173 \pm 0.002$ & $0.0163 \pm 0.0004$ \\
\hline
\end{tabular}

Table 5: Count rate comparison with previous detectors, measured in [counts $/ \mathrm{keV} / \mathrm{kg} / \mathrm{y}$ ]. Here CCVR values are after polonium subtraction, as described in 4.1 Errors are statistical.
${ }^{6}$ In some of CCVR1 measurements ( $4 \%$ of the CCVR lifetime) only two ${ }^{306}$ facing crystals were active. The extrapolation of M2 counts to M1 is performed ${ }^{307}$ considering that the coincidence analysis is sensitive only to 2 over 12 faces. $\quad 308$

It is interesting to compare CCVRs rates with the ones ob-283 tained with previous detectors, like TTT [11] or Cuoricino [7]. This comparison is shown in Table 5, where the M1 and M2 count rates for CCVRs are after polonium subtraction, as $\mathrm{de}_{-}{ }^{284}$ scribed in 4.1 the continuum region $(2700,3200) \mathrm{keV}$ and $(3400,3900) \mathrm{keV}_{286}$ excluding the ${ }^{190} \mathrm{Pt}$ energy region of $(3200,3400) \mathrm{keV}$. It can be 287 inferred that : coincidence rate is compatible within $1.8 \sigma$ with the cor- ${ }^{289}$ responding TTT value.

- in the region $(4000,5000) \mathrm{keV}$, as already measured in ${ }^{291}$ the TTT run, a reduction in the count rate with respect to ${ }^{292}$ Cuoricino is observed.

- in the region $(5000,6000) \mathrm{keV}$ a comparison is not possi- ${ }^{294}$ ble since CCVR is affected by the high rate of ${ }^{210} \mathrm{Po}$. This ${ }^{295}$ is due to the fact that in CCVRs only recently grown crys $^{296}$ tals are measured, unlike TTT or Cuoricino. the TTT run, a reduction in the count rate with respect $\mathrm{to}_{300}$ Cuoricino is present. However, the CCVR count rate is $s_{301}$ greater than the TTT value, probably because of the pres- ${ }_{302}$ ence of mis-identified pile-up (in M1) or coincidences (in M2) with ${ }^{210} \mathrm{Po}$ events, extending above $6000 \mathrm{keV}$. This ${ }^{303}$ contribution should decay away with ${ }^{210} \mathrm{Po}$. 06
The notation $(2700,3900)$ refers to the combination of both ${ }^{285}$

- in the region $(2700,3900) \mathrm{keV}$ the CCVRs anti- ${ }^{288}$

- in the region $(6000,8000) \mathrm{keV}$, as already measured in ${ }_{200}$ (a)
A more detailed evaluation of the comparison can be found in [11].

\section{Results on bulk contaminations of CUORE crystals}

Bulk contaminations in CUORE crystals are expected from:

- ${ }^{210} \mathrm{Po}$, as a result of the chemical affinity between polonium and tellurium;

- natural contaminants, like ${ }^{238} \mathrm{U}$ and ${ }^{232} \mathrm{Th}$ with their radioactive chains;

- ${ }^{210} \mathrm{~Pb}$, as a result of the deposition of lead nuclei produced by ${ }^{222} \mathrm{Rn}$ decays, during the crystal handling in free atmosphere;

- ${ }^{190} \mathrm{Pt}$, due to the fact that platinum is used in several phases of the crystal production cycle. Platinum crucibles are used for the calcination of $\mathrm{TeO}_{2}$ powder used for the crystal growth and the growth crucibles are made of platinum foil. The central part of the as-grown crystal ingot is selected for the CUORE crystals in order to avoid the risk of platinum contamination on the surface of the crystal due to possible diffusion during the growth process. Possible Pt contaminations are therefore in the bulk of the CUORE crystals.

For ${ }^{210}$ Po the activity is determined from a fit to the anticoincidence rate vs. time behaviour (see Sec. 5.1).

For ${ }^{238} \mathrm{U}$ and ${ }^{232} \mathrm{Th}$ (see Sec. 5.2), the limit on the level of contamination is determined from the intensities of the $\alpha$ peaks in the anti-coincidence spectrum of all crystals, or from integrals centered at the peak position if the peaks are not visible. 


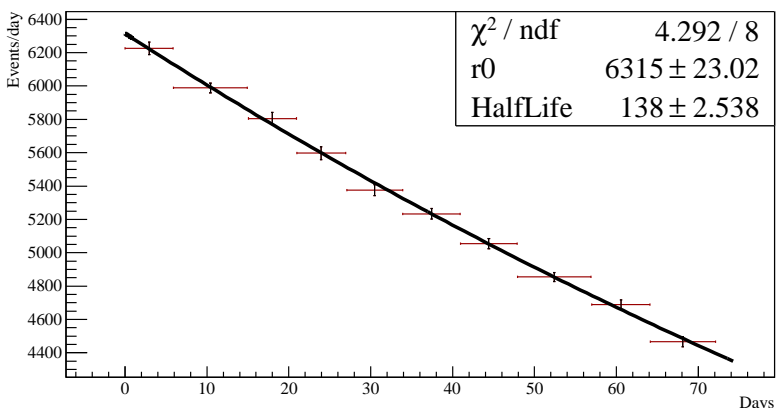

Figure 3: Global rate of ${ }^{210}$ Po events over time for each CCVR1. The fit result is overlayed.

This is because an $\alpha$ decay from bulk contamination releases the entire $\mathrm{Q}$-value of the reaction ( $\alpha$ energy + nuclear recoil) in a single crystal.

For ${ }^{210} \mathrm{~Pb}$, the limit on bulk contamination is determined from a fit in the energy region $(40,60) \mathrm{keV}$ in a subset of CCVRs data with high statistics and low threshold (see Sec.6).

\section{1. ${ }^{210}$ Po bulk activity}

The activity of ${ }^{210} \mathrm{Po}$ can be measured from the intensity of $5407.5 \mathrm{keV} \alpha$ line in the anti-coincidence spectrum.

The plot in Fig. [3] shows the global rate of ${ }^{210} \mathrm{Po}$ events over time for CCVR1. For each channel the ${ }^{210}$ Po events are selected in a $\pm 20 \mathrm{keV}$ window around the energy of the $\alpha$ line.

The units on the $\mathrm{x}$-axis are days since the start of the first background measurement. Each point represents a group of measurements whose livetime is at least 5 days.

The horizontal error bars indicate the beginning and the end of each group of runs, they are for visualization only.

Each point is corrected with the corresponding rate-based efficiency, calculated with the equation (1). A larger dead time window (9 seconds instead of 7 seconds) compared to the rest of the analysis is used, to be more conservative in the removal of pile-up pulses.

The fit function is a pure exponential:

$$
r(t)=r_{0} e^{\left(-\ln 2 t / T_{1 / 2}\right)}
$$

where $r_{0}$ is the rate at the beginning of the measurement and $T_{1 / 2}$ is the ${ }^{210}$ Po half-life. The half-life of the exponential decay has been evaluated for all CCVRs and it is shown in Tab 6 it is in good agreement $(1 \sigma)$ with the half-life of ${ }^{210} \mathrm{Po}(138.38 \mathrm{~d}){ }^{351}$ This indicates that the ${ }^{210} \mathrm{Po}$ contamination is out of equilibrium and it is not being fed by ${ }^{210} \mathrm{~Pb}$.

From the value of $r_{0}$ returned by the fit for each CCVR and for each crystal, the ${ }^{210}$ Po activity at the beginning of the mea- ${ }^{353}$ surement is extracted as follows:

$$
A[\mathrm{~Bq} / \mathrm{kg}]=\frac{r_{0}}{86400[\mathrm{~s} / \mathrm{day}] m[\mathrm{~kg}]}
$$

where $m$ is the crystal mass.

Knowing the time elapsed since the "crystal birth date" (growth completed, before the cut and shape) and the start of $f_{360}$ the measurement, the ${ }^{210} \mathrm{Po}$ activity at production is computed. 361

\begin{tabular}{|c|c|}
\hline Run & $\begin{array}{c}\text { Half-life } \\
\text { [days] }\end{array}$ \\
\hline \hline CCVR1 & $138.0 \pm 2.5$ \\
CCVR2 & $132.3 \pm 14.7$ \\
CCVR3 & $139.4 \pm 6.3$ \\
CCVR4 & $137.8 \pm 11.5$ \\
CCVR5 & $136.6 \pm 9.1$ \\
\hline
\end{tabular}

Table 6: ${ }^{210}$ Po half-life for all CCVRs. The activity has been fitted using an exponential function. All the values are consistent (within $1 \sigma$ ) with 138.38 days.

The ${ }^{210}$ Po activities for each CCVR and for all crystals are reported in Table 7. The results for crystals 007 and 011, measured first in CCVR1 and again in CCVR2, are consistent.

All crystals tested in the first five CCVRs are well below the limit of $0.1 \mathrm{~Bq} / \mathrm{kg}$ imposed to the crystal producers.

\begin{tabular}{|c|c|c|}
\hline CCVR & Crystal & ${ }^{210}$ Po activity [Bq $\left./ \mathrm{kg}\right]$ \\
\hline \hline 1 & 041 & $0.0257 \pm 0.0001$ \\
1 & 011 & $0.0510 \pm 0.0005$ \\
1 & 039 & $0.0229 \pm 0.0001$ \\
1 & 007 & $0.0414 \pm 0.0004$ \\
\hline \hline 2 & 076 & $0.021 \pm 0.004$ \\
2 & 011 & $0.07 \pm 0.02$ \\
2 & 096 & $0.055 \pm 0.006$ \\
2 & 007 & $0.047 \pm 0.015$ \\
\hline \hline 3 & 190 & $0.0078 \pm 0.0005$ \\
3 & 236 & $0.0159 \pm 0.0005$ \\
3 & 180 & $0.0203 \pm 0.0008$ \\
3 & 229 & $0.0283 \pm 0.0008$ \\
\hline \hline 4 & 340 & $0.032 \pm 0.004$ \\
4 & 313 & $0.005 \pm 0.001$ \\
4 & 354 & $0.039 \pm 0.004$ \\
4 & 380 & $0.040 \pm 0.004$ \\
\hline \hline 5 & 455 & $0.019 \pm 0.002$ \\
5 & 416 & $0.024 \pm 0.003$ \\
5 & 436 & $0.032 \pm 0.003$ \\
5 & 421 & $0.020 \pm 0.003$ \\
\hline
\end{tabular}

Table 7: ${ }^{210} \mathrm{Po}$ activity at production time for all CCVRs crystals.

\subsection{U/Th bulk contaminations}

${ }^{238} \mathrm{U}$ and ${ }^{232} \mathrm{Th}$ bulk contaminations are evaluated from the anti-coincidence sum spectrum of all CCVRs. For each of the peaks reported in Table 8, the number of counts (corrected by the efficiency of the event-based cuts from Table 3) within an energy window of $\pm 6 \sigma$ around the $\alpha$ Q-value is estimated. A $\sigma$ of $2.2 \mathrm{keV}$, corresponding to the average between the values of the ${ }^{210}$ Po peak $\sigma$ s for each CCVR, weighted by the corresponding lifetime of that run (see Table 9 for details) is used. Using the Bayesian approach, the upper limits $N_{u}$ at $90 \%$ C.L. 


\begin{tabular}{|c|c|c|c|c|}
\hline Chain & Nuclide & $\begin{array}{c}\text { Energy } \\
{[\mathrm{keV}]}\end{array}$ & Half-life & $\begin{array}{c}N_{u} \\
\text { 90\% C.L. }\end{array}$ \\
\hline \hline${ }^{238} \mathrm{U}$ & ${ }^{238} \mathrm{U}$ & 4270.0 & $4.47 \mathrm{E}+09 \mathrm{y}$ & 11.7 \\
& ${ }^{234} \mathrm{U}$ & 4858.8 & $2.45 \mathrm{E}+05 \mathrm{y}$ & 20.8 \\
& ${ }^{230} \mathrm{Th}$ & 4770.0 & $7.54 \mathrm{E}+04 \mathrm{y}$ & 24.9 \\
& ${ }^{226} \mathrm{Ra}$ & 4870.6 & $1599 \mathrm{y}$ & 30.0 \\
& ${ }^{218} \mathrm{Po}$ & 6114.7 & $3.05 \mathrm{~min}$ & 7.4 \\
\hline${ }^{232} \mathrm{Th}$ & ${ }^{232} \mathrm{Th}$ & 4082.8 & $1.4 \mathrm{E}+10 \mathrm{y}$ & 5.9 \\
& ${ }^{212} \mathrm{Bi}$ & 6207.1 & $60.55 \mathrm{~min}$ & 13.5 \\
\hline
\end{tabular}

Table 8: $90 \%$ C.L. limits on the number of events ascribed to several nuclides from uranium and thorium decay chain. For each nuclide is also shown the Q-value and the half-life of the $\alpha$ decay.

\begin{tabular}{|c|c|c|}
\hline Run & $\begin{array}{c}\text { Livetime } \\
\text { [days] }\end{array}$ & $\begin{array}{c}\sigma \\
{[\mathrm{keV}]}\end{array}$ \\
\hline \hline CCVR1 & 59.9 & 1.6 \\
CCVR2 & 19.4 & 1.4 \\
CCVR3 & 43.05 & 2.2 \\
CCVR4 & 25.8 & 3.5 \\
CCVR5 & 30.3 & 2.8 \\
\hline
\end{tabular}

Table 9: Summary of CCVRs energy resolutions, evaluated with a gaussian fit of the $5407.5 \mathrm{keV}$ peak from ${ }^{210} \mathrm{Po}$. The average value of $2.2 \mathrm{keV}$, used for ${ }_{392}$ the global analysis is obtained averaging the resolution of each CCVR with the corresponding livetime.

are obtained, assuming 0 expected background counts and a flat ${ }^{396}$ prior for the signal [12] (see Table 8).

The upper limit on the activity for each nuclide is calculated 398 using the following formula:

$$
A_{u}[\mathrm{~Bq} / \mathrm{kg}]=\frac{N_{u}}{\varepsilon_{M C} T[\mathrm{~s}] m[\mathrm{~kg}] \Gamma}
$$

where $\varepsilon_{M C}$ is the Monte Carlo detection efficiency, $T$ the ${ }^{403}$ CCVRs livetime, $m$ the crystal mass and $\Gamma$ the branching ratio ${ }^{404}$ of the nuclide. In this analysis a total containment of the anti- ${ }^{405}$ coincidence events in the crystals is assumed $\left(\varepsilon_{M C}=1\right)$. Results ${ }^{406}$ are shown in Table 10.

The upper limit for confidence level of $90 \%$ for U/Th bulk ${ }^{408}$ contaminations are then calculated in the hypothesis of secular ${ }^{409}$ equilibrium within the uranium and thorium decay chains. Re- ${ }^{410}$ sults for the total CCVRs data are shown in the last column of ${ }^{411}$ Table 10.

In the most conservative approach, the bulk contamination ${ }^{413}$ limit on ${ }^{238} \mathrm{U}$ and ${ }^{232} \mathrm{Th}$ is set considering the most active nu- ${ }^{414}$ clide for each chain. The upper limit at $90 \%$ C.L. for uranium ${ }^{415}$ and thorium bulk contamination are:

$$
\begin{aligned}
{ }^{238} \mathrm{U} & <5.3 \cdot 10^{-14}[\mathrm{~g} / \mathrm{g}] \\
{ }^{232} \mathrm{Th} & <2.1 \cdot 10^{-13}[\mathrm{~g} / \mathrm{g}]
\end{aligned}
$$

Both values of the upper limits are within the contract specifica-421 tion of $3 \cdot 10^{-13}[\mathrm{~g} / \mathrm{g}]$. For the ${ }^{238} \mathrm{U}$ decay chain, the contribution ${ }_{422}$ from ${ }^{210} \mathrm{~Pb}$ is treated separately (see Sec.6).

It is important to stress the fact that the above upper limits 424 are calculated under the hypothesis that the observed counts for ${ }_{425}$

\begin{tabular}{|c|c|c|c|}
\hline Chain & Nuclide & $\begin{array}{c}\text { Upper limit } \\
{[\mathrm{Bq} / \mathrm{kg}]}\end{array}$ & $\begin{array}{c}\text { Upper limit } \\
{[\mathrm{g} / \mathrm{g}]}\end{array}$ \\
\hline \hline${ }^{238} \mathrm{U}$ & ${ }^{238} \mathrm{U}$ & $2.5 \mathrm{E}-07$ & $2.0 \mathrm{E}-14$ \\
& ${ }^{234} \mathrm{U}$ & $4.7 \mathrm{E}-07$ & $3.6 \mathrm{E}-14$ \\
& ${ }^{230} \mathrm{Th}$ & $5.7 \mathrm{E}-07$ & $4.4 \mathrm{E}-14$ \\
& ${ }^{226} \mathrm{Ra}$ & $6.7 \mathrm{E}-07$ & $5.3 \mathrm{E}-14$ \\
& ${ }^{218} \mathrm{Po}$ & $1.6 \mathrm{E}-07$ & $1.3 \mathrm{E}-14$ \\
\hline${ }^{232} \mathrm{Th}$ & ${ }^{232} \mathrm{Th}$ & $1.3 \mathrm{E}-07$ & $3.1 \mathrm{E}-14$ \\
& ${ }^{212} \mathrm{Bi}$ & $8.4 \mathrm{E}-07$ & $2.1 \mathrm{E}-13$ \\
\hline
\end{tabular}

Table 10: Upper limits at $90 \%$ C.L. on the activity and on the bulk contamination of uranium and thorium decay chains in the hypothesis of secular equilibrium.

each nuclide are entirely due to a bulk contamination of that nuclide. This is a conservative hypothesis since there is not a clear indication (for example a line) that such a bulk contamination actually exists and that the observed counts are not due to background of some other origin.

\section{6. ${ }^{210} \mathrm{~Pb}$ activity}

During the production of CUORE crystals great care is devoted in order to minimize the exposure of the crystals to free atmosphere, to avoid contamination of radon and its daughters. Nevertheless, a small ${ }^{210} \mathrm{~Pb}$ contamination (bulk or surface) might occur. This contamination can be very dangerous because, even if the soft beta $(16.96 \mathrm{keV}$ and $63.5 \mathrm{keV})$ and $\gamma(46.5 \mathrm{keV})$ radiation from ${ }^{210} \mathrm{~Pb}$ is generally self absorbed, the induced bremsstrahlung of the daughter ${ }^{210} \mathrm{Bi}$ can give rise to a continuum up to $1.16 \mathrm{MeV}$. Moreover, the $\alpha$ decay of the daughter ${ }^{210} \mathrm{Po}$ can contribute to the continuum background in the double beta decay energy region.

In CCVRs crystals the ${ }^{210} \mathrm{~Pb}$ contamination cannot be estimated from ${ }^{210} \mathrm{Po}$, because this contamination is out of equilibrium (see Sec. 5.1). The only available signature is a combination of a beta spectrum (end point $16.96 \mathrm{keV}$ ) with a deexcitation energy of $46.5 \mathrm{keV}$ (most of the cases through a conversion electron). This produces a broad signature in the energy region $(40,60) \mathrm{keV}$, whose shape depends on the location of the $\mathrm{Pb}$ contamination.

Several simulations are performed both for a bulk contamination and for a surface contamination with exponential density profile [9] and contamination depth varying from $0.01 \mu \mathrm{m}$ to $10 \mu \mathrm{m}$. Even if, for the sake of simplicity, a common energy threshold of $50 \mathrm{keV}$ is set for all CCVRs, there are subset of data where the threshold can be set to a lower value in order to look for this signature. CCVR1 data are used because they struk a balance between high livetime (CCVR1 has the highest statistic) and good bolometer performances. Only for CCVR1 data, the analysis is repeated with an energy threshold of $40 \mathrm{keV}$. The anti-coincidence spectrum between 40 and $60 \mathrm{keV}$ is fitted with an exponentially decreasing background and allowing the presence of a ${ }^{210} \mathrm{~Pb}$ spectrum with shape taken from Monte Carlo simulation. The free parameters of the fit are the two parameters of the exponential background and the total number 

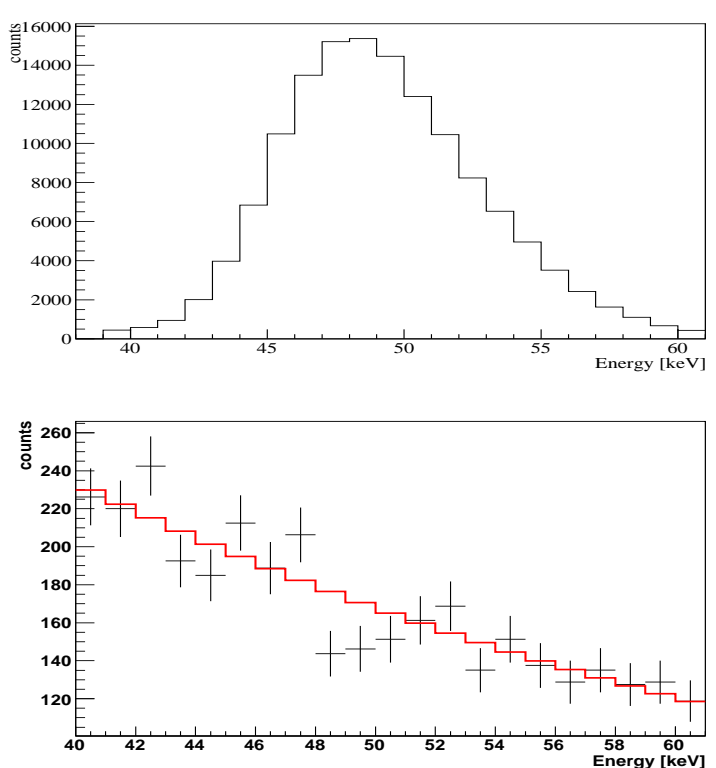

Figure 4: Top: Monte Carlo simulation of a ${ }^{210} \mathrm{~Pb}$ bulk contamination in ${ }^{45}$ CCVR1. Bottom: CCVR1 low energy anti-coincidence spectrum. The energy threshold is set to $40 \mathrm{keV}$. The red line represents the fit to the spectrum with an exponential background and allowing the presence of a ${ }^{210} \mathrm{~Pb}$ bulk contamination with the above shape. No hint of such contamination is found.

of counts from ${ }^{210} \mathrm{~Pb}$. The fit is repeated for each Monte Carlo signature. The number of ${ }^{210} \mathrm{~Pb}$ events is compatible with zero within the error for all signatures. An example of Monte Carlo spectrum for a ${ }^{210} \mathrm{~Pb}$ bulk contamination and the corresponding fit to the experimental spectrum is shown in Fig.4.

The upper limit at $90 \%$ C.L. on the number of ${ }^{210} \mathrm{~Pb}$ counts is $N_{u}=1.644 \sigma$, where $\sigma$ is the error on the number of counts returned from the fit. The upper limit on bulk contamination is computed using Eq. 5. The upper limit on surface contamina ${ }^{-460}$ tion is extracted using the following formula:

$$
A_{u}\left[\mathrm{~Bq} / \mathrm{cm}^{2}\right]=\frac{N_{u}}{\varepsilon_{M C} T[\mathrm{~s}] S\left[\mathrm{~cm}^{2}\right]}
$$

\begin{tabular}{|c|c|}
\hline contamination & Upper limit 90\% C.L. \\
\hline \hline bulk & $3.3 \mathrm{E}-06$ \\
surf. $0.01 \mu \mathrm{m}$ & $9.8 \mathrm{E}-07$ \\
surf. $0.1 \mu \mathrm{m}$ & $3.8 \mathrm{E}-08$ \\
surf. $0.2 \mu \mathrm{m}$ & $2.2 \mathrm{E}-08$ \\
surf. $1 \mu \mathrm{m}$ & $9.2 \mathrm{E}-09$ \\
surf. $5 \mu \mathrm{m}$ & $5.6 \mathrm{E}-09$ \\
surf $10 \mu \mathrm{m}$ & $4.9 \mathrm{E}-09$ \\
\hline
\end{tabular}

Table 11: Upper limits on the activity of bulk and surface contamination of ${ }^{210} \mathrm{~Pb}$. Bulk contamination is given in $[\mathrm{Bq} / \mathrm{kg}]$, surface contaminations are given in $\left[\mathrm{Bq} / \mathrm{cm}^{2}\right]$.

\subsection{U/Th surface contaminations}

The M2sum spectrum in Fig. 2] shows a huge background for energies above the polonium $\alpha$ line due to random coincidences between ${ }^{210} \mathrm{Po}$ and low energy events. At a closer inspection (see Fig. 5), the peaks originated by the sum of a $5407.5 \mathrm{keV} \alpha$ line and a low energy $\gamma$ line arising from Te metastable isotopes are clearly visible. See also Table 12 for details.

\begin{tabular}{|c|c|c|}
\hline Te isotope & $\begin{array}{c}\text { Energy } \\
{[\mathrm{keV}]}\end{array}$ & $\begin{array}{c}\text { Energy + 5407.5 } \\
{[\mathrm{keV}]}\end{array}$ \\
\hline \hline${ }^{127} \mathrm{Te}$ & 88.3 & 5495.8 \\
${ }^{129} \mathrm{Te}$ & 105.5 & 5513 \\
${ }^{125} \mathrm{Te}$ & 144.8 & 5552.3 \\
${ }^{123} \mathrm{Te}$ & 247.5 & 5655 \\
${ }^{121} \mathrm{Te}$ & 294 & 5701.5 \\
\hline
\end{tabular}

Table 12: Energy of the gamma lines from Tellurium metastable isotopes and corresponding sum energy when in coincidence with a ${ }^{210}$ Po event.

Because of the presence of these coincidences, only nuclides with a Q-value lower than $5407.5 \mathrm{keV}$ (see Table 13) are used for surface contamination analysis. ${ }^{218} \mathrm{Po}$ and ${ }^{212} \mathrm{Bi}$ are therefore discared. The scatter plot of M2 events is shown in Fig.6 The shadowed region contains the events with a total energy within 4 and $5 \mathrm{MeV}$.

For each nuclide listed in Table 13, the number of counts is computed from the M2sum energy spectrum in an energy window of $\pm 6 \sigma$ around the Q-value and divided by $\varepsilon^{2}$ (where $\varepsilon$ is the efficiency of the event-based cuts of Table 3), since two coincident events have independent probabilities of passing the cuts. In Table 13 are shown the corresponding upper limits at 90\% C.L., computed using the Bayesian approach with 0 expected background counts and a flat prior for the signal [12].

Monte Carlo spectra for ${ }^{238} \mathrm{U}$ and ${ }^{232} \mathrm{Th}$ contamination on the crystal surface are generated with exponential profile and various penetration lengths. For each contamination depth, the containment efficiency is calculated in a $\pm 6 \sigma$ interval around the Q-value of each nuclide both for the M2sum spectrum and for the M1 spectrum. Results are shown in Fig. 7. For penetration length of 0.01 and $0.1 \mu \mathrm{m}$ the higher containment efficiency comes from the M2sum spectrum, whereas for depths of 1,5 and $10 \mu \mathrm{m}$ this arises from the M1 spectrum. The efficiency 

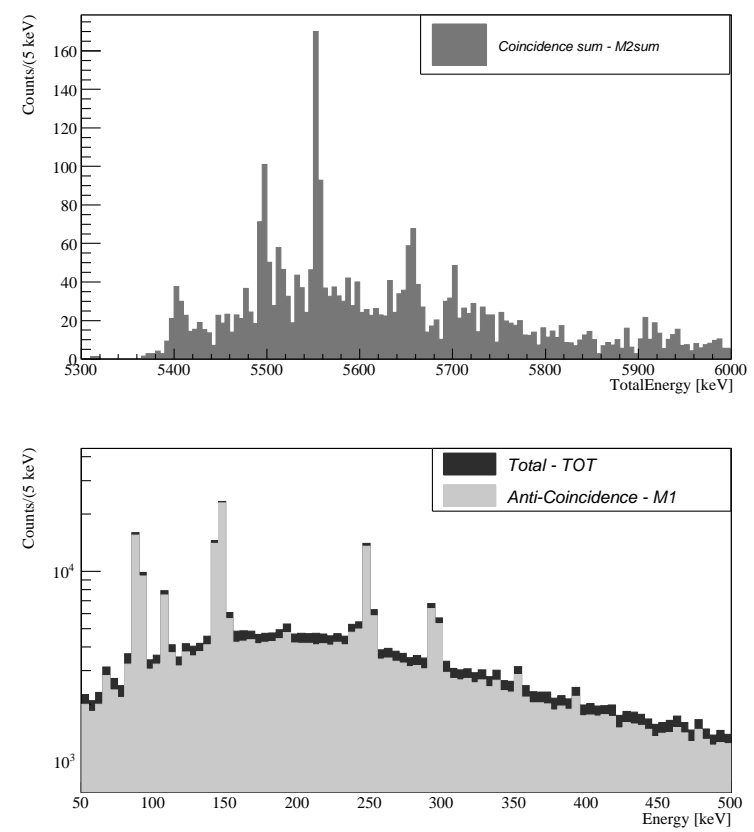
nated by the coincidence of the $5407.5 \mathrm{keV} \alpha$ line and a low energy $\gamma$ line from 490 Te metastable isotopes are clearly visible. Bottom: total and anti-coincidence spectra in the region 0-500 keV: low energy lines from Te metastable isotopes are clearly visible.

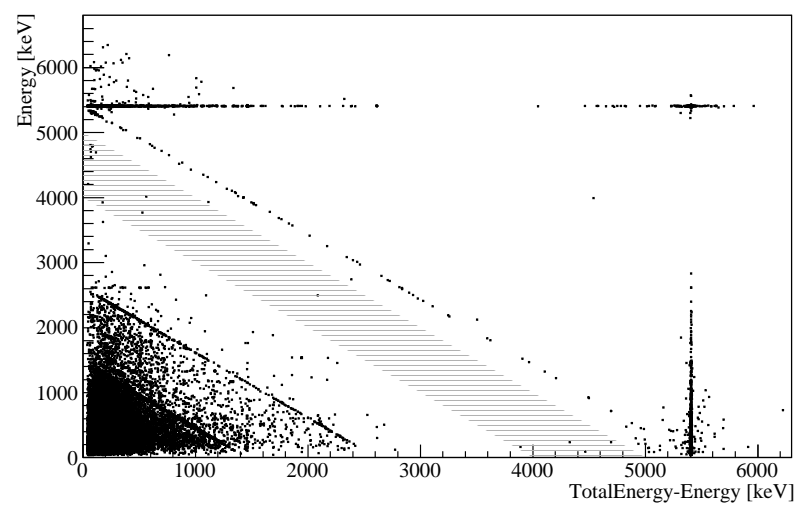

Figure 6: Scatter plot of $\mathrm{M} 2$ events. The shadowed region contains events with ${ }^{508}$ total energy between 4 and $5 \mathrm{MeV}$, used for surface analysis.

\begin{tabular}{|c|c|c|c|c|}
\hline Chain & Nuclide & $\begin{array}{c}\text { Half-life } \\
{[y]}\end{array}$ & $\begin{array}{c}\text { Energy range } \\
{[\mathrm{keV}]}\end{array}$ & $\begin{array}{c}N_{u} \\
90 \% \text { C.L. }\end{array}$ \\
\hline \multirow[t]{4}{*}{${ }^{238} \mathrm{U}$} & $\overline{238 \mathrm{U}}$ & $\overline{4.47 \mathrm{E}+09}$ & $4257-4283$ & 4.6 \\
\hline & ${ }^{234} \mathrm{U}$ & $2.45 \mathrm{E}+05$ & $4845-4871$ & 4.6 \\
\hline & ${ }^{230} \mathrm{Th}$ & $7.54 \mathrm{E}+04$ & $4757-4783$ & 4.6 \\
\hline & ${ }^{226} \mathrm{Ra}$ & 1599 & $4857-4883$ & 10.5 \\
\hline${ }^{232} \mathrm{Th}$ & ${ }^{232} \mathrm{Th}$ & $1.4 \mathrm{E}+10$ & $4069-4095$ & 2.3 \\
\hline
\end{tabular}

Table 13: Upper limits at $90 \%$ C.L. on the number of counts ascribed to several ${ }^{517}$ nuclides from uranium and thorium decay chain from the M2sum spectrum.518 For each nuclide, the half-life of the $\alpha$ decay is also shown.

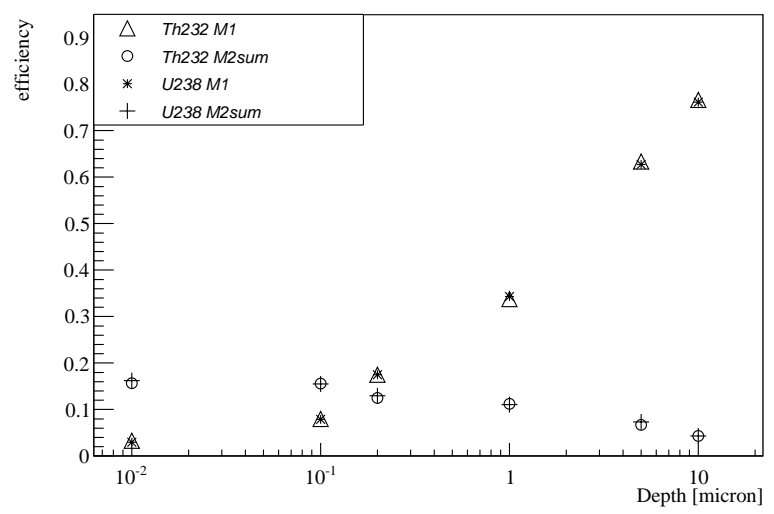

Figure 7: For each penetration length, the containment efficiency of surface events is computed for both M1 and M2sum Monte Carlo spectra. and

For the ${ }^{232} \mathrm{Th}$ chain there is only one useable line for this analysis, that is the one from ${ }^{232} \mathrm{Th}$. This means that there is no way of testing the portion of the chain below ${ }^{220} \mathrm{Rn}$ and take into account a possible non-equilibrium of the chain, as done for the bulk contamination.

The results for surface contaminations are shown in Table 14 culated under the hypothesis that the observed counts for each nuclide are evaluated using Eq. 6 where $N_{u}$ is the $90 \%$ C.L. upper limit on the number of observed events from the M2sum or the M1 spectrum (see Table 13 for the M2sum counts and Table 8 for the M1 counts) and $\varepsilon_{M C}$ is the Monte Carlo average efficiency defined in eq. 2 for the corresponding spectrum (M2sum or M1) and for the given signature. The confidence intervals for surface activity contaminations are calculated for both the M2sum and M1 spectra, normalizing each signature with the corresponding Monte Carlo efficiency. The signature giving the most stringent result is taken into account.

For surface contaminations of $0.01,0.1$ and $0.2 \mu \mathrm{m}$ depths, the most stringent limits come from the M2sum spectrum. For the remaining depths $(1,5$ and $10 \mu \mathrm{m})$ the surface activity reduces practically to a bulk activity and the M1 signature produces the most stringent limits (see Table 8 for details).

For the ${ }^{238} \mathrm{U}$ chain, the surface contamination for 2 peaks is

- ${ }^{238} \mathrm{U}$, the chain parent;

- ${ }^{226} \mathrm{Ra}$, the most active line both in M1 and M2sum spectra.

The contribution from ${ }^{210} \mathrm{~Pb}$ is treated separately (see Sec. 6). 
nuclide are entirely due to a surface contamination of that nuclide in the corresponding depth. Again, this is a very conservative hypothesis.

\begin{tabular}{|c|c|c|}
\hline Depth & Nuclide & $\begin{array}{c}\text { Upper limit 90\% C.L. } \\
{\left[\mathrm{Bq} / \mathrm{cm}^{2}\right]}\end{array}$ \\
\hline \hline $0.01 \mu \mathrm{m}$ & ${ }^{238} \mathrm{U}$ & $3.1 \mathrm{E}-09$ \\
& ${ }^{226} \mathrm{Ra}$ & $6.3 \mathrm{E}-09$ \\
& ${ }^{232} \mathrm{Th}$ & $1.6 \mathrm{E}-09$ \\
\hline \hline $0.2 \mu \mathrm{m}$ & ${ }^{238} \mathrm{U}$ & $3.8 \mathrm{E}-09$ \\
& ${ }^{226} \mathrm{Ra}$ & $7.6 \mathrm{E}-09$ \\
& ${ }^{232} \mathrm{Th}$ & $2.0 \mathrm{E}-09$ \\
\hline \hline $1 \mu \mathrm{m}$ & ${ }^{238} \mathrm{U}$ & $3.7 \mathrm{E}-09$ \\
& ${ }^{226} \mathrm{Ra}$ & $8.9 \mathrm{E}-09$ \\
& ${ }^{232} \mathrm{Th}$ & $1.9 \mathrm{E}-09$ \\
\hline \hline $5 \mu \mathrm{m}$ & ${ }^{238} \mathrm{U}$ & $2.0 \mathrm{E}-09$ \\
& ${ }^{226} \mathrm{Ra}$ & $5.4 \mathrm{E}-09$ \\
& ${ }^{232} \mathrm{Th}$ & $1.0 \mathrm{E}-09$ \\
\hline \hline $10 \mu \mathrm{m}$ & ${ }^{238} \mathrm{U}$ & $1.7 \mathrm{E}-09$ \\
& ${ }^{226} \mathrm{Ra}$ & $4.4 \mathrm{E}-09$ \\
& ${ }^{232} \mathrm{Th}$ & $8.3 \mathrm{E}-10$ \\
\hline
\end{tabular}

Table 14: Upper limits at $90 \%$ C.L. for surface contamination, for different ${ }^{556}$ penetration length values. See text for details on the calculation of confidence intervals.

\section{Extrapolation to CUORE background}

In order to evaluate the contribution to the CUORE background arising from crystal contamination, a simulation both for bulk and surface contamination reproducing the CUORE geometry and studying the contribution to the double beta decay (DBD) energy region is performed.

Being interested in a conservative upper limit to the CUORE background, in this extrapolation it has been assumed that CUORE crystals will have the same activity as crystals tested in CCVRs. However, when CUORE will start the data taking, most of the ${ }^{210}$ Po (and other short lived states) will have decayed and the efficiency for pile-up rejection will be increased.

\subsection{Background from bulk contamination}

The CUORE geometrical efficiency for a uniform bulk contamination of crystals in ${ }^{210} \mathrm{~Pb},{ }^{238} \mathrm{U}$ and ${ }^{232} \mathrm{Th}$ is estimated via Monte Carlo, and the corresponding CUORE background the DBD energy region (Q-value $\pm 30 \mathrm{keV}$ ) is calculated using the formula:

$$
\mathrm{bkg}_{\mathrm{CUORE}}=\frac{A_{\mathrm{CCVR}-\mathrm{bulk}} \varepsilon_{M C}^{\mathrm{CUORE}-\mathrm{bulk}}}{\Delta E}
$$

where $A_{\mathrm{CCVR}}$-bulk are the values of ${ }^{210} \mathrm{~Pb},{ }^{238} \mathrm{U}$ and ${ }^{232} \mathrm{Th}$ activities from Tables 10 and $11, \varepsilon_{M C}^{\mathrm{CUORE}-\text { bulk }}$ is evaluated from Monte Carlo simulation and $\Delta \mathrm{E}=60 \mathrm{keV}$.

The results are shown in Table 15

In the most conservative approach, considering the most ac-561 tive line $\left({ }^{212} \mathrm{Bi}\right)$, the following upper limit to the CUORE back-562 ground at the DBD energy due to bulk contamination of crystals 563 is set: $1.1 \cdot 10^{-4}$ counts $/ \mathrm{keV} / \mathrm{kg} / \mathrm{y}$.

\begin{tabular}{|c|c|c|}
\hline chain & Nuclide & $\begin{array}{c}\text { Upper Limit 90\% C.L. } \\
\text { [counts/keV/kg/y] }\end{array}$ \\
\hline \hline & ${ }^{210} \mathrm{~Pb}$ & $2.6 \mathrm{E}-05$ \\
\hline${ }^{238} \mathrm{U}$ & ${ }^{238} \mathrm{U}$ & $8.1 \mathrm{E}-07$ \\
& ${ }^{234} \mathrm{U}$ & $1.4 \mathrm{E}-06$ \\
& ${ }^{230} \mathrm{Th}$ & $1.7 \mathrm{E}-06$ \\
& ${ }^{226} \mathrm{Ra}$ & $2.1 \mathrm{E}-06$ \\
& ${ }^{218} \mathrm{Po}$ & $5.1 \mathrm{E}-07$ \\
\hline${ }^{232} \mathrm{Th}$ & ${ }^{232} \mathrm{Th}$ & $1.7 \mathrm{E}-05$ \\
& ${ }^{212} \mathrm{Bi}$ & $1.1 \mathrm{E}-04$ \\
\hline
\end{tabular}

Table 15: Extrapolation to CUORE background from CCVRs bulk contamination limits from Tabs. 10 and 11

\subsection{Background from surface contamination}

In a similar way, the CUORE geometrical efficiency for a surface contamination for several depths is estimated. The corresponding CUORE background is extrapolated using the formula:

$$
\mathrm{bkg}_{\mathrm{CUORE}}=\frac{A_{\mathrm{CCVR}-\text { surf }} \varepsilon_{M C}^{\mathrm{CUORE}-\text { surf }} S}{\Delta E M_{\mathrm{CUORE}}}
$$

where $A_{\mathrm{CCVR}-\text { surf }}$ are the surface contamination values from Ta-

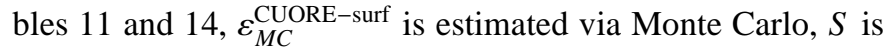
the surface of the CUORE crystals, $\Delta \mathrm{E}=60 \mathrm{keV}$ and $M_{\mathrm{CUORE}}$ $=0.75 \cdot 988 \mathrm{~kg}$.

\begin{tabular}{|c|c|c|}
\hline Depth & Nuclide & $\begin{array}{c}\text { Upper limit 90\% C.L. } \\
\text { [counts/keV/kg/y] }\end{array}$ \\
\hline \hline $0.01 \mu \mathrm{m}$ & ${ }^{210} \mathrm{~Pb}$ & $1.6 \mathrm{E}-03$ \\
& ${ }^{238} \mathrm{U}$ & $8.9 \mathrm{E}-05$ \\
& ${ }^{226} \mathrm{Ra}$ & $1.8 \mathrm{E}-04$ \\
& ${ }^{232} \mathrm{Th}$ & $1.8 \mathrm{E}-05$ \\
\hline \hline $0.2 \mu \mathrm{m}$ & ${ }^{210} \mathrm{~Pb}$ & $2.3 \mathrm{E}-04$ \\
& ${ }^{238} \mathrm{U}$ & $3.9 \mathrm{E}-04$ \\
& ${ }^{226} \mathrm{Ra}$ & $7.7 \mathrm{E}-04$ \\
& ${ }^{232} \mathrm{Th}$ & $1.5 \mathrm{E}-04$ \\
\hline \hline $1 \mu \mathrm{m}$ & ${ }^{210} \mathrm{~Pb}$ & $3.8 \mathrm{E}-04$ \\
& ${ }^{238} \mathrm{U}$ & $1.2 \mathrm{E}-03$ \\
& ${ }^{226} \mathrm{Ra}$ & $2.9 \mathrm{E}-03$ \\
& ${ }^{232} \mathrm{Th}$ & $4.4 \mathrm{E}-04$ \\
\hline \hline $5 \mu \mathrm{m}$ & ${ }^{210} \mathrm{~Pb}$ & $4.3 \mathrm{E}-04$ \\
& ${ }^{238} \mathrm{U}$ & $1.3 \mathrm{E}-03$ \\
& ${ }^{226} \mathrm{Ra}$ & $3.4 \mathrm{E}-03$ \\
& ${ }^{232} \mathrm{Th}$ & $4.7 \mathrm{E}-04$ \\
\hline \hline $10 \mu \mathrm{m}$ & ${ }^{210} \mathrm{~Pb}$ & $2.9 \mathrm{E}-04$ \\
& ${ }^{238} \mathrm{U}$ & $7.1 \mathrm{E}-04$ \\
& ${ }^{226} \mathrm{Ra}$ & $1.9 \mathrm{E}-03$ \\
& ${ }^{232} \mathrm{Th}$ & $3.0 \mathrm{E}-04$ \\
\hline
\end{tabular}

Table 16: Extrapolation to CUORE background from CCVRs surface contamination limits from Tables 11 and 14

The results are shown in Table 16 Based on these results, in the most conservative approach, a crystal surface contamination in CUORE within the limits estimated from CCVRs data, would yield a rate in the DBD energy region lower than 


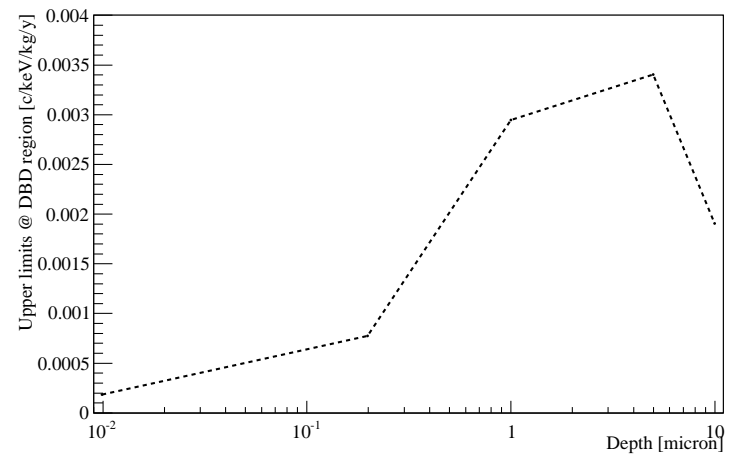

Figure 8: For each penetration depth, the upper limits at 90\% C.L. for the rate at the DBD energy region are plotted. The values are computed on ${ }^{226} \mathrm{Ra}_{617}$ line, and are due only to surface crystals contaminations.

$5.5 \cdot 10^{-3}$ counts $/ \mathrm{keV} / \mathrm{kg} / \mathrm{y}$. This value corresponds to the worst case: a contamination in ${ }^{238} \mathrm{U}$ and ${ }^{232} \mathrm{Th}$ at $5 \mu \mathrm{m}$ and a contamination in ${ }^{210} \mathrm{~Pb}$ at $0.01 \mu \mathrm{m}$. This result is strictly dependent on the density profile assumed for the contaminants distribution, as can be seen in Fig. 8, where the upper limit at the 90\% C.L. for the rate in the DBD energy region (computed on ${ }^{226} \mathrm{Ra}$ line) is plotted in function of contamination depth.

\section{Conclusions}

The CUORE Crystal Validation Runs (CCVRs) have been carried out since the end of 2008 at the Gran Sasso National Laboratories, in order to test the performances and the radiopurity of the $\mathrm{TeO}_{2}$ crystals produced at SICCAS (Shanghai Institute of Ceramics, Chinese Academy of Sciences) for the CUORE experiment.

In this work the results of the first 5 validation runs, are presented. The items under analysis are the background rate, the bulk and surface contamination from several nuclides and the extrapolation to the CUORE background.

The CCVRs background rate shows a reduction with respect to the one measured in CUORICINO in all the energy regions considered. The bulk activity of ${ }^{210} \mathrm{Po}$ is measured to be within the limit specified in the contract with the crystals producer. No indication of a bulk contamination from uranium and thorium decay chains, as well as from ${ }^{210} \mathrm{~Pb}$ (out of equilibrium), is found. The upper limits are calculated to be within the contract specification. No indication of a surface contamination from uranium and thorium decay chains, as well as from ${ }^{210} \mathrm{~Pb}$ (out of equilibrium), is found. Upper limits at $90 \%$ C.L. are calculated for surface contamination from several nuclides and for different contamination depths.

An extrapolation to CUORE background from both bulk and surface contaminations is performed, in the most conservative assumption that the CCVRs observed background is entirely due to the bulk and the surface contamination respectively. Considering only the contribution from bulk and surface crystals contaminations, the following upper limits to the CUORE background index in the energy region around the Q-value of the neutrinoless double beta decay of ${ }^{130} \mathrm{Te}$ are calculated:
$1.1 \cdot 10^{-4}$ counts $/ \mathrm{keV} / \mathrm{kg} / \mathrm{y}$ and $5.5 \cdot 10^{-3}$ counts $/ \mathrm{keV} / \mathrm{kg} / \mathrm{y}$ respectively.

\section{References}

[1] R. Ardito et al., arXiv:hep-ex/0501010

[2] C. Arnaboldi et al., Nucl. Instr. Meth. A 518 (2004) 775.

[3] C. Arnaboldi et al., Journal of Crystal Growth 312 (2010) 2999.

[4] E.E. Haller et al., edited by R. D. Larrabee (Plenum Press, New York, 1984) 21.

[5] C. Arnaboldi et al., Phys. Rev. C 78 (2008) 035502.

[6] S. Pirro, Nucl. Instr. Meth. A 559 (2006) 672.

[7] E. Andreotti et al., Astr. Phys. 34 (2011) 822.

[8] E. Gatti and P.F. Manfredi, Rivista del Nuovo Cimento 9 (1986) 1.

[9] C. Bucci et al., Eur. Phys. J A 41 (2009) 155.

[10] M. Pavan et al., Eur. Phys. J A 36 (2008) 159.

[11] CUORE Coll. "Three Tower Test: copper surface treatments for low background DBD experiments with $\mathrm{TeO}_{2}$ bolometers." Paper in preparation.

[12] K. Nakamura et al. (Particle Data Group), J. Phys. G 37, 075021 (2010) 